\title{
Activity of Daily Living Trajectories Surrounding Acute Hospitalization of Long-Stay Nursing Home Residents
}

\author{
Robin L. Kruse, PhD, * Gregory F. Petroski, PhD, ${ }^{\dagger}$ David R. Mehr, MD, MS, * \\ Jane Banaszak-Holl, $\mathrm{PhD},{ }^{+\$}$ and Orna Intrator, $\mathrm{PhD}^{\mathrm{I} * *}$
}

OBJECTIVES: To explore patterns of change in nursing home (NH) residents' activities of daily living (ADLs), particularly surrounding acute hospital stays.

DESIGN: Longitudinal study using Medicare and Minimum Data Set (MDS) assessments.

SETTING: National sample of long-stay NH residents.

PARTICIPANTS: NH residents who were hospitalized for the seven most-common inpatient diagnoses $(\mathrm{N}=40,128)$. Each hospital admission was at least 90 days after any prior hospitalization and had at least two preceding MDS assessments.

MEASUREMENTS: The MDS ADL long-form score, a simple sum of seven self-care variables coded from 0 (independent) to 4 (totally dependent) was used to indicate resident ADL function. Scores ranged from 0 to 28 , with higher scores indicating greater impairment. A linear mixed model describing ADL trajectories was jointly estimated with time-to-event models for mortality and hospital readmission.

RESULTS: Before hospitalization, the most common trajectory was stable $(53.7 \%)$, with $27.5 \%$ of residents worsening and $18.8 \%$ improving. ADL function after hospital discharge was most often characterized as stable $(43.1 \%)$ or worsening $(39.2 \%)$. Mortality $(20.3 \%)$ was higher for those with worsening prehospital ADL function $(28.9 \%)$ than for those with stable $(19.1 \%)$ or improving $(11.3 \%)$ trajectories. Hospital diagnosis was associated with amount of ADL worsening and rate of subsequent $\mathrm{ADL}$ change. Most residents with the best initial function continued to worsen after hospital discharge. Cognitive

From the *Department of Family and Community Medicine, and 'Biostatistics and Research Design Unit, University of Missouri,

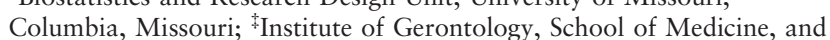
${ }^{\S}$ Department of Health Management and Policy, School of Public Health, University of Michigan, Ann Arbor, Michigan; "Providence Veterans Affairs Medical Center, and * Center for Gerontology and Health Care Research, Brown University, Providence, Rhode Island.

Address correspondence to Robin L. Kruse, MA306 Medical Sciences Building, Department of Family and Community Medicine, School of Medicine, University of Missouri, Columbia, MO 65212.

E-mail: kruser@health.missouri.edu

DOI: $10.1111 /$ jgs. 12511 impairment was associated with poorer ADL function and accelerated worsening of ADLs.

CONCLUSION: For many long-stay NH residents, substantial and sustained ADL worsening accompanies acute hospitalization, so acute hospitalization presents an opportunity to revisit care goals; the results of the current study can help inform decision-making. J Am Geriatr Soc 61:1909-1918, 2013.

Key words: nursing homes; activities of daily living; hospitalization; Minimum Data Set

$\mathbf{I}_{\mathrm{p}}^{\mathrm{n}}$ ndependence in activities of daily living (ADLs) - basic personal care activities such as dressing, eating, and moving about-is positively associated with quality of life. ${ }^{1,2}$ Conversely, ADL impairments are strongly associated with poorer physical health, ${ }^{3}$ hospital admission, ${ }^{4,5}$ higher cost of medical care, ${ }^{6}$ and death. ${ }^{3,4,7-11}$ Thus, a nursing home (NH) resident's ADL status and likely pattern of change over time, or trajectory, are important considerations for determining care priorities. Because hospitalization is often associated with at least a transient decline in ADL, ${ }^{12-18}$ residents' prehospital ADL trajectories may act as "vital signs" that can help determine appropriate care plans in the hospital and after discharge. ${ }^{19}$

Many NH residents are long-stay residents with low potential for returning home; ${ }^{20,21}$ their care needs and ADL status will probably change over time. Fifteen percent to $25 \%$ of long-stay residents may be hospitalized over a period of 6 months. ${ }^{22-24}$ Residents' medical conditions can contribute to ADL decline, ${ }^{5,25}$ as can complications associated with hospitalization caused by prolonged immobilization, iatrogenic problems, sensory deprivation, and poor nutritional intake. ${ }^{12,13}$ Hospitalization can result in pressure sores, infections, and delirium ${ }^{12,15,26,27}$ and may increase the risk of subsequent cognitive and functional decline. ${ }^{17}$

For some, hospitalization indicates a poor prognosis. For example, individuals with severe dementia who are 
hospitalized for pneumonia or a hip fracture have high 6-month mortality. ${ }^{28}$ For others, return from the hospital might require physical therapy and rehabilitation. Despite the potential value of ADL trajectories, few studies have explored ADL change in $\mathrm{NH}$ residents, particularly as hospitalization influences them. ${ }^{29,30}$

Most previous research has considered change in ADLs between two fixed times (e.g., baseline and 90 days), often dichotomizing change as declined or not. ${ }^{15,16,31-33}$ Depending on the span between time points and whether they encompass an acute event, substantial ADL changes could be missed. Although several studies have reported an association between ADL decline and hospitalization, most used such a before-and-after comparison. It is unclear whether residents' ADL function was declining before hospitalization and how much recovery, if any, followed hospital discharge. Moreover, existing information provides little insight into the functional prognosis of residents returning to a $\mathrm{NH}$ after hospitalization.

In a study that examined $\mathrm{ADL}$ trajectories of $\mathrm{NH}$ residents before and after initiating dialysis, ${ }^{34}$ predialysis ADL scores were worsening slightly. In the 3 months after dialysis initiation, median ADL scores for survivors worsened; only $13 \%$ of residents maintained their predialysis function. A random-effects model showed accelerating ADL decline surrounding dialysis initiation, followed by stabilization between months 1 and 4 and continued worsening after month 4 . The current study was designed to use a similar approach in a broader sample of $\mathrm{NH}$ residents.

To better understand the ADL trajectories of residents who returned to a $\mathrm{NH}$ after a hospitalization, ADL trajectories of long-stay $\mathrm{NH}$ residents before and after acute hospitalization were examined to determine the effect of prehospital ADL trajectories on posthospitalization outcomes and to determine how resident characteristics and diagnosis influence this relationship. It was hypothesized that residents with worsening ADL trajectories before hospital admission would be more likely to die or exhibit worsening ADL trajectories after hospitalization than those with stable or improving trajectories. It was further hypothesized that residents with good ADL function before hospitalization would return to their prehospital level more rapidly after hospital discharge than residents with poorer baseline function.

\section{METHODS}

\section{Analytical Cohort}

Minimum Data Set (MDS) assessments and Medicare inpatient records were obtained from the Centers for Medicare and Medicaid Services to assemble a cohort of long-stay residents who had had an acute hospitalization (Figure 1). Residents younger than 67 as of January 1, 2006, residents with any health maintenance organization membership during 2006 to 2007 (because health maintenance organizations do not report hospital data), residents not appearing in the beneficiary summary files for 2006 to 2007, residents with date discrepancies (e.g., multiple dates of death), residents lacking Part A coverage for either year, and residents with more than 15 acute hospitalizations in 2006 and 2007 were excluded.

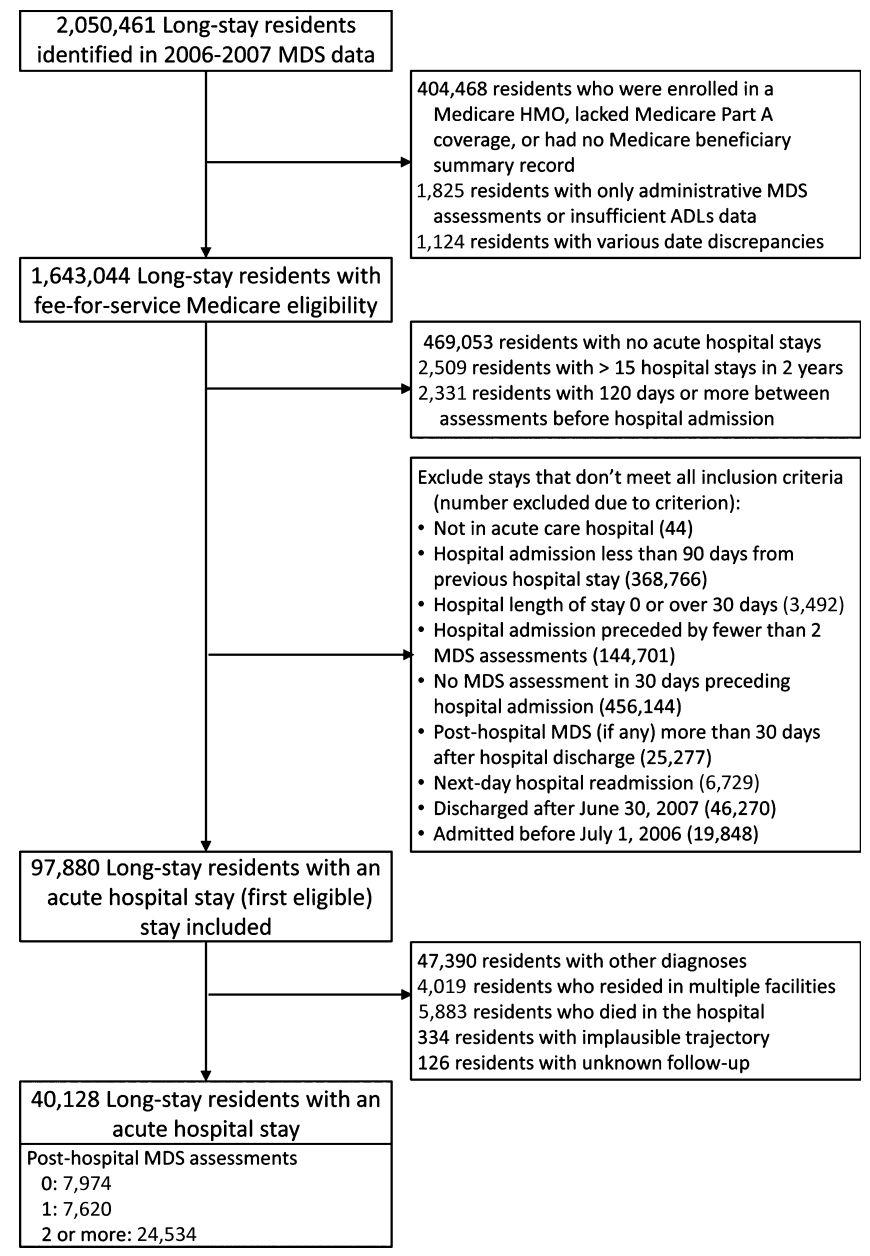

Figure 1. Flow diagram showing derivation of cohort of longstay nursing home residents. $\mathrm{MDS}=$ Minimum Data Set; $\mathrm{ADLs}=$ activities of daily living; $\mathrm{HMO}=$ health maintenance organization.

Each resident's first stay in an acute care hospital of 30 or fewer days in length and having at least two preceding MDS assessments with ADL data, one of which was within the 30 days preceding hospitalization to help define the trajectory before hospitalization, was included. Because hospitalization is associated with substantial ADL change, only hospital stays that were 90 days or more from any prior stay were included to minimize the effect of the prior hospital stay. To allow for 6 months of MDS assessments before and after hospitalization, stays with an admission date on or after July 1, 2006, and a discharge date before July 1, 2007, were included. Because the focus was on posthospital outcomes, residents who died in the hospital were excluded. It was also required that residents reside in one facility for all included assessments.

All of a resident's MDS assessments during the 6 months before and after hospitalization except those occurring before a preceding hospital stay or after a subsequent stay were included. MDS assessments typically occur quarterly, with at least one full (more detailed) assessment occurring annually. Additional assessments may occur when a resident has a substantial change in condition or receives Medicare-paid skilled nursing care; timing and number of posthospital assessments differ according to 
state. The longitudinal ADL record for each resident was terminated at 180 days of follow-up or at time of death or hospital readmission if either event occurred within the 180-day window. Multiple MDS assessments from the same day, assessments with target dates before 2006, and "impossible" assessments dated in the middle of long hospital stays were also excluded. Hospital stays were excluded when a subsequent stay occurred within 1 day of discharge.

The multilevel Clinical Classification Software (http:// www.hcup-us.ahrq.gov/toolssoftware/ccs/ccs.jsp) was used to group primary hospital diagnoses into categories. Because the goal was to compare the effects of specific hospital diagnoses on posthospital ADL trajectories, residents with the seven most common hospital diagnoses were included-pneumonia, septicemia, congestive heart failure, hip fracture, renal failure, stroke, and urinary tract infection. These diagnoses accounted for $51.6 \%$ of the sample and included chronic and acute conditions, common infections, and conditions often associated with rehabilitation. Because additional diagnoses occur in diminishing frequency while adding to model complexity, they were excluded.

\section{Outcome Variable}

ADL function was measured using the MDS ADL longform score, ${ }^{35}$ a simple sum of seven self-care variables (bed mobility, self-transfer, locomotion on unit, dressing, eating, toileting, and personal hygiene) from Section G of MDS 2.0. The weighted kappas for the seven component activities were $>0.75$, indicating excellent reliability. ${ }^{35}$ Internal consistency of the scale is also high $(\alpha=0.94){ }^{35}$ Item scores varied from 0 (independence) to 4 (total dependence). Item scores of 8 (activity did not occur during the entire week), were recoded to 4 before summing. The total score ranged from 0 to 28 , with 0 indicating complete independence and 28 indicating complete dependence in all seven activities.

Deteriorating ADL function was defined as a gain of 3 or more points per year, whereas losing 3 or more points per year delineated improvement. ${ }^{16}$ Change of three points or more indicates a substantial change in assistance, denoting an increase or decrease in the need for cueing or supervision of three ADL activities or the addition or loss of physical assistance for one activity. The Cognitive Performance Scale (CPS) score (0 (intact cognition) to 6 (severe impairment)) was calculated at the time of the first MDS assessment. $^{36}$

\section{Resident Characteristics}

MDS records and beneficiary summary files were used to determine resident demographic characteristics. When sources disagreed, the beneficiary summary file prevailed. The earliest date a diagnosis was assigned to a resident was derived by combining data from Medicare claims, MDS assessments, and the Chronic Condition Warehouse data furnished by the Centers for Medicare and Medicaid Services. This date of earliest diagnosis was used to determine diagnoses present at the time of hospital admission, which were used to calculate the Charlson Index.

\section{Statistical Analysis}

All analyses were performed in SAS version 9.3 (SAS Institute, Inc., Cary, NC). The overall strategy was to determine how hospital diagnosis and length of stay modified ADL trajectory. Because MDS assessments are typically performed quarterly, time was measured in fractions of quarters before hospital admission or after discharge, with baseline covariates measured at the earliest included prehospital assessment. ADL trajectories were modeled using a linear mixed-effects model. Separate covariance structures allowed the person-specific random slopes and intercepts to vary over pre- and posthospital periods.

Loss to follow-up due to death or hospital readmission is a significant feature of this cohort. Death and readmission represent potentially nonrandom selection mechanisms, limiting the estimation of trajectories to healthy survivors. ${ }^{37}$ Simultaneously modeling the longitudinal outcome (ADL) and the time to dropout can limit the biasing effects of nonrandom dropout, ${ }^{38,39}$ so ADL trajectory, survival time, and time to readmission were jointly modeled. ${ }^{40}$ Death and readmission were each modeled using a separate log-normal regression. The time-to-event models for readmission and mortality shared a subject-specific intercept and slope for posthospital ADL ability. Model fitting was done using the NLMIXED procedure. A more-detailed description of the model appears in Appendix S1. Parameter estimates were used to determine expected trajectories for groups of residents with specific characteristics.

Prior research has found associations between ADL change and baseline ADL function, ${ }^{15,16,41}$ prior ADL loss, ${ }^{19}$ cognition, ${ }^{14,41,42}$ and acute illness indicators. ${ }^{15,16}$ The desire to include important covariates was balanced with their availability on quarterly MDS forms and an overall strategy of model parsimony. To explore the effects of good, moderately impaired, and severely impaired baseline ADL function, residents' initial ADL scores were divided into three categories (0-4, 5-23, 24-28). Resident's initial ADL category, age, sex, Charlson Comorbidity Index ${ }^{43}$ (based on prehospital diagnoses), and baseline CPS score $^{36}$ were included as covariates during the pre- and posthospital periods. Primary hospital diagnosis and length of stay were included as covariates only during the posthospital period. Interactions between time and initial ADL category, CPS score, length of stay, and primary hospital diagnosis were also entered into the model to determine their effect on the rate of change in ADL score. The ADL and CPS interaction terms were included across both time periods, whereas the interactions between time and hospital diagnosis and between time and length of stay were present only after hospitalization.

To evaluate the effects of prehospital slope on posthospital outcomes, residents' person-specific predicted slopes were characterized as worsening, stable, or improving. Chi-square analysis was used to determine whether prehospital slope was associated with 30-day mortality or readmission.

\section{RESULTS}

Of an initial cohort of slightly more than 2 million longstay residents, 97,880 residents meeting initial selection 
criteria were identified (Figure 1). Of these, 47,390 $(48.4 \%)$ residents who were not hospitalized for one of the included diagnoses, 5,883 $(6.0 \%)$ who died in the hospital, and 4,019 (4.1\%) assessed in multiple facilities were excluded; 126 residents $(0.1 \%)$ who lacked any follow-up data and $334(0.3 \%)$ with erratic and seemingly implausible ADL patterns were also excluded, leaving 40,128 $(41.0 \%)$ long-stay residents who survived hospitalization.

Most $(70.5 \%)$ residents were female, $51.5 \%$ were aged 85 and older, $84.6 \%$ were white, and $10.9 \%$ were black (Table 1). Demographic characteristics were similar to those of the initial sample of 2,050,461 long-stay residents, of which $71.3 \%$ were female, $85.0 \%$ were white, and $9.9 \%$ were black. A smaller proportion of all long-stay residents $(45.8 \%)$ were aged 85 and older. Residents who were least impaired at baseline (ADL score $<5$ ) were most likely to have no or mild cognitive impairment $(70.9 \%)$, whereas those with severely impaired ADL function usually had moderately impaired $(41.6 \%)$ or severely impaired $(46.8 \%)$ cognition. Median number of MDS assessments before and after hospitalization was 2 (mean: 2.8 prehospital and 2.5 posthospital).

Using person-specific predicted slopes, the most common prehospital ADL trajectory was stable $(53.7 \%)$; $27.5 \%$ of residents had a worsening trajectory, and $18.8 \%$ were improving. Prehospital ADL trajectory was associated with baseline ADL score. Residents with the best prehospital ADL score had a higher proportion of worsening trajectories $(61.4 \%)$ than those who were moderately $(31.1 \%)$ or severely impaired $(2.4 \%)$.

The most common comorbidities before hospital admission were diabetes mellitus $(38.7 \%)$, congestive heart failure $(32.6 \%)$, and chronic obstructive pulmonary disease $(31.2 \%)$. Most residents $(60.4 \%)$ had a Charlson Index ${ }^{43}$ of 2 or higher. Hospital admission was usually because of infection, most commonly pneumonia (33.5\%), septicemia $(17.0 \%)$, and urinary tract infection $(15.4 \%)$. Infections were more prevalent in residents with severely impaired baseline ADL function than in those with low to intermediate baseline ADL function. Conversely, congestive heart failure, hip fracture, renal failure, and stroke were more common in residents with less ADL impairment.

Mortality (20.3\%) and readmission (19.6\%) were common in the 30 days after hospital discharge (Table 2). Using person-specific predicted ADL slopes, mortality was higher for those with worsening prehospital function $(28.9 \%)$ than for those with stable $(19.1 \%)$ or improving $(11.3 \%)$ function $(P<.001)$. Readmission varied less, affecting $18.5 \%$ of residents with worsening prehospital ADL function and approximately $20.0 \%$ of residents who were improving or stable before hospitalization $(P=.001)$. After hospital discharge, stable trajectories were the most common $(43.1 \%)$, followed by worsening $(39.2 \%)$ and improving $(17.7 \%)$ trajectories.

Parameter estimates for the mixed model are shown in Table 3. Factors that affect ADL level are the main-effect estimates that represent the change in ADL score associated with that variable (intercepts). Factors that affect the rate of change are estimates of a variable's interactions with time (slopes). Several factors were associated with ADL score, including female sex and CPS. Before and after hospitalization, each point increase in CPS was associated with a 0.94-point worsening in ADL score. CPS score was also associated with rate of ADL change; each point increase in CPS score was associated with a 0.24-point worsening in ADL score per quarter, or a 0.96-point worsening per year. High or low baseline ADL scores were also associated with rate of change in ADL function. Overall mean ADL score changed at the rate of -0.41 points per quarter $(-1.64$ points per year), indicating improvement. In addition to the overall mean, good initial ADL function was associated with a 1.39-point worsening in ADL score per quarter, whereas poor baseline function was associated with a -1.09-point change (improvement) per quarter. Given the other variables in the model, age did not appear to be associated with ADL score $(P=.86)$.

Primary hospital diagnosis and length of stay were associated with change in posthospital ADL score and change in rate of posthospital ADL recovery. On average, hip fracture added the most to the ADL score (7.65), followed by stroke (6.53), renal failure (3.41), septicemia (2.97), urinary tract infection (2.75), pneumonia (2.74), and congestive heart failure (2.64). Only hip fracture was associated with ADL improvement after hospital discharge $(-1.39$ points per quarter). The remaining diagnoses were associated with worsening posthospital ADL function.

Because of the interaction terms in the model, the combined effects of diagnosis and baseline ADL function on resident ADL trajectories can be viewed only by modeling groups of residents with chosen characteristics. Figure 2 presents the expected ADL trajectories based on the model presented in Table 3 for residents with two diagnoses: hip fracture and pneumonia. Residents with modal characteristics were used for the illustration: aged 85 and older, female, moderate baseline ADL impairment, moderate cognitive impairment (CPS score $=3$ ), Charlson Index of 2, and a 5 -day hospital stay. The $y$-axis has been reversed so that worsening scores decline visually. In the prehospital period, ADL function worsens slowly. Because the hospital diagnosis is not present during the prehospital period, the prehospital trajectories are identical for both diagnoses. Surrounding the acute hospitalization, ADL function worsens precipitously, with the amount of change equal to the diagnosisspecific intercepts in Table 3. The effect of this substantial change in ADL score over a short period in time is a trajectory that appears discontinuous. For example, hip fracture is associated with a 7.65-point change and pneumonia with a 2.74-point change. After hospital discharge, residents who had a hip fracture improve, whereas those who were hospitalized for pneumonia (and the other diagnoses) continue to worsen. Because each additional point on the CPS scale shifts the ADL score upward and increases the slope of the trajectory, residents with poor cognition have poorer ADL function at all times than those with good cognition, as well as ADL function that worsens at a greater rate (not illustrated).

Many of the same factors were associated with readmission and mortality (Table 4). Time was log-transformed for these time-to-event models, so the parameter estimates were exponentiated and are interpreted as multiplicative factors (values $>1$ indicate longer time to event). For example, women were less likely to be readmitted and had lower mortality $(24 \%$ longer time to readmission and $15 \%$ longer time to death) than men. Age lengthened time 
Table 1. Characteristics of Long-Stay Nursing Home Residents with a Minimum Data Set (MDS) Assessment within 30 Days of Hospital Admission Grouped According to Baseline Activity of Daily Living (ADL) Score

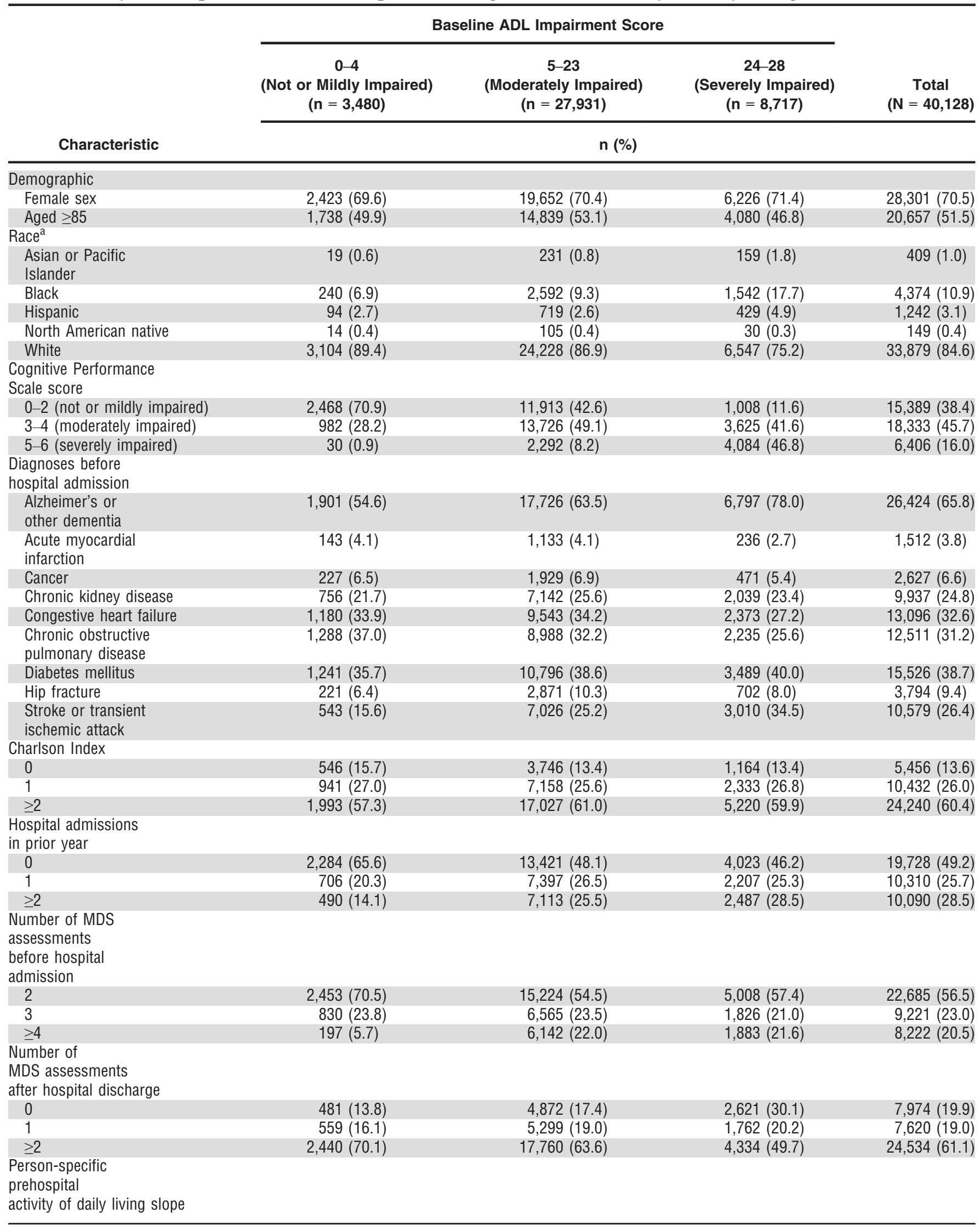


Table 1. (Contd.)

\begin{tabular}{|c|c|c|c|c|}
\hline \multirow[b]{2}{*}{ Characteristic } & $\begin{array}{c}0-4 \\
\text { (Not or Mildly Impaired) } \\
(\mathrm{n}=3,480)\end{array}$ & $\begin{array}{c}5-23 \\
\text { (Moderately Impaired) } \\
(n=27,931)\end{array}$ & $\begin{array}{c}24-28 \\
\text { (Severely Impaired) } \\
(n=8,717)\end{array}$ & $\begin{array}{c}\text { Total } \\
(\mathrm{N}=40,128)\end{array}$ \\
\hline & \multicolumn{4}{|c|}{ n (\%) } \\
\hline Worsening & $2,137(61.4)$ & $8,677(31.1)$ & $209(2.4)$ & $11,023(27.5)$ \\
\hline \multicolumn{5}{|l|}{ Hospital diagnosis } \\
\hline Congestive heart failure & $604(17.4)$ & 3,433 (12.3) & $468(5.4)$ & $4,505(11.2)$ \\
\hline Hip fracture & 657 (18.9) & 3,107 (11.1) & $210(2.4)$ & $3,974(9.9)$ \\
\hline Pneumonia & $1,081(31.1)$ & $8,991(32.2)$ & $3,358(38.5)$ & $13,430(33.5)$ \\
\hline Renal failure & $274(7.9)$ & $2,101(7.5)$ & $516(5.9)$ & $2,891(7.2)$ \\
\hline
\end{tabular}

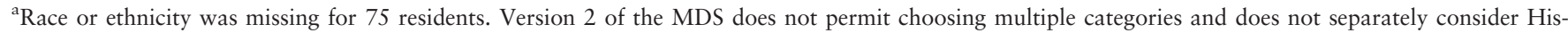
panic ethnicity.

Table 2. Outcomes After Hospital Discharge for Long-Stay Nursing Home Residents ${ }^{\mathrm{a}}$

Person-Specific Prehospital ADL Slope

\begin{tabular}{|c|c|c|c|c|c|}
\hline \multirow{2}{*}{$\begin{array}{l}\text { Postdischarge } \\
\text { Outcome }\end{array}$} & $\begin{array}{l}\text { Improving } \\
(\mathrm{n}=7,550)\end{array}$ & $\begin{array}{c}\text { Stable } \\
(\mathrm{n}=21,555)\end{array}$ & $\begin{array}{l}\text { Worsening } \\
(n=11,023)\end{array}$ & $\begin{array}{c}\text { Total } \\
(\mathrm{N}=40,128)\end{array}$ & $P$-Value ${ }^{\mathrm{b}}$ \\
\hline & \multicolumn{5}{|c|}{ n (\%) } \\
\hline nission within 30 days & $1,512(20.0)$ & 4,333 (20.1) & $2,036(18.5)$ & 7,881 (19.6) & .001 \\
\hline within 30 days & $853(11.3)$ & $4,122(19.1)$ & 3,188 (28.9) & $8,163(20.3)$ & $<.001$ \\
\hline $\begin{array}{l}\text { ר-specific posthospital } \\
\text { lope }^{c}\end{array}$ & & & & & \\
\hline roving & 2,626 (34.8) & $3,100(14.4)$ & $1,376(12.5)$ & 7,102 (17.7) & $<.001$ \\
\hline ole & 3,381 (44.8) & $10,984(51.0)$ & $2,911(26.4)$ & $17,276(43.1)$ & \\
\hline rsening & $1,543(20.4)$ & $7,471(34.7)$ & $6,736(61.1)$ & $15,750(39.2)$ & \\
\hline
\end{tabular}

${ }^{a} 40,128$ residents who survived their hospital stay and were not lost to follow-up.

${ }^{\mathrm{b}} \mathrm{P}$-value for chi-square test of outcomes according to prehospital person-specific slope.

${ }^{c}$ The model calculates a predicted value for all residents. Residents who have no posthospital activity of daily living (ADL) data are assigned the group mean slope for individuals with their characteristics (e.g., sex, baseline ADL, diagnosis).

to readmission, but residents aged 85 and older had a higher death rate ( $26 \%$ fewer days to death) than younger residents. Residents with higher CPS were slightly less likely to be readmitted and more likely to die. Residents with hospital diagnoses other than hip fracture were more likely to be readmitted, as were residents with higher Charlson scores and longer stays. Septicemia and stroke were associated with higher mortality than congestive heart failure, whereas hip fracture and urinary tract infection were associated with lower mortality.

\section{DISCUSSION}

ADL trajectories of a cohort of long-stay $\mathrm{NH}$ residents surrounding an acute hospitalization were examined. Overall, ADL function worsened between pre- and posthospital assessments, and for most residents, the posthospital trajectory continued this trend. Trajectories differed depending on prehospital ADL score, cognition, and primary hospital diagnosis. Residents with significant cognitive impairment had worse ADL trajectories after hospitalization than residents with better cognition. Understanding the more-limited potential for recovery of residents with significant cognitive impairment is important in helping families and facilities develop meaningful care plans for these residents, including decisions about limiting aggressive treatment.

It was surprising to see that, on average, residents with good baseline function did not improve after acute hospitalization. Except for residents hospitalized for hip fracture, these residents worsened after hospital discharge, causing the hypothesis that they would return to baseline ADL level more quickly than more impaired residents to be rejected. As shown in Table 1, 61\% of residents with good initial ADL function had declining trajectories before hospitalization. Thus, their higher probability of decline after hospitalization continued their prehospital trend. The initially declining trajectories of these higher-functioning 


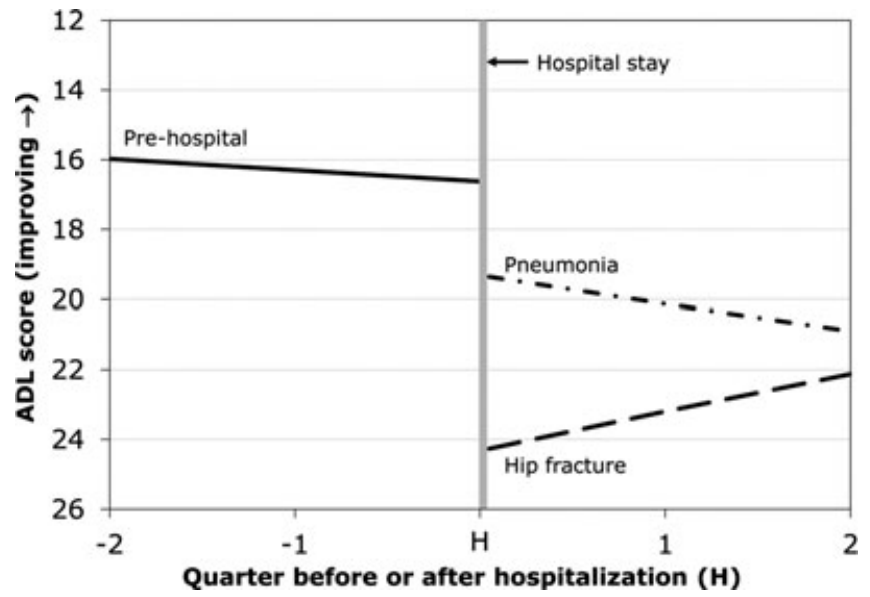

Figure 2. Average activity of daily living (ADL) trajectories predicted by regression model (Table 3 ) for nursing home residents hospitalized for hip fracture or pneumonia. Because the primary hospital diagnosis is not present before the acute event, the prehospital trajectory is identical for the two diagnoses. Precipitous worsening surrounding the acute hospitalization follows the slow prehospital worsening in ADL function. The amount of worsening varies according to diagnosis and is equal to the intercepts in Table 3; hip fracture was associated with a 7.65-point change and pneumonia with a 2.74-point change. On average, individuals with hip fracture improve after hospital discharge, whereas residents hospitalized for pneumonia (and the other diagnoses) continue to worsen. For purposes of illustration, the modal characteristics of the population were used for other resident characteristics: aged $\geq 85$, female, moderately impaired cognition (Cognitive Performance Scale score 3), moderate baseline ADL impairment (baseline ADL score 5-23), Charlson Comorbidity Index of 2, and hospital length of stay of 5 days.

residents perhaps reflects that, in many cases, they had some limitations in areas other than ADL function that led to long-term NH care.

Primary hospital diagnosis had a strong effect on the shift in ADL score upon hospitalization and the rate of change in ADL score after hospital discharge. Residents hospitalized for hip fracture or stroke, conditions often associated with a need for rehabilitation, exhibited the largest change in ADL score, worsening more than 6 points on the 28-point scale immediately after hospitalization. Residents hospitalized for congestive heart failure had the smallest change in ADL score. Longer hospital stays, which are probably a proxy for severity of the acute illness, were also associated with worse posthospital ADL scores. After jointly modeling ADL trajectories with mortality and readmission, only the group of residents with poor initial function exhibited any posthospital ADL recovery, and the recovery rate for most diagnoses was modest.

Few other studies have used mixed models to study ADL trajectories of $\mathrm{NH}$ residents. One study ${ }^{42}$ used MDS data to study ADL trajectories of residents who had been in a facility for 1 year or longer. They excluded residents with severe cognitive impairment (CPS score $=6$ ) or complete eating dependency at admission. Representing ADL function as a sum of five items to form a scale from 0 to 20, mean ADL score increased 0.07 points per month, indicating slow worsening. In contrast, the current study
Table 3. Parameter Estimates for the Activity of Daily Living (ADL) Trajectory Portion of the Joint Model $(\mathrm{N}=40,128$ Subjects $)$

\begin{tabular}{|c|c|c|}
\hline Variable & $\begin{array}{c}\text { Parameter Estimate } \\
\text { (95\% Confidence } \\
\text { Interval) }\end{array}$ & $P$-Value \\
\hline $\begin{array}{l}\text { Baseline ADL } \\
\text { level (intercept) }\end{array}$ & $13.5(13.4-13.6)$ & $<.001$ \\
\hline \multicolumn{3}{|l|}{$\begin{array}{l}\text { Factors that } \\
\text { affect ADL level } \\
\text { (intercept) across } \\
\text { both time periods }\end{array}$} \\
\hline Female & $0.17(0.09-0.26)$ & $<.001$ \\
\hline Age $>85$ & $-0.01(-0.08-0.07)$ & .86 \\
\hline $\begin{array}{l}\text { Charlson } \\
\text { Comorbidity Index }\end{array}$ & $0.05(0.03-0.08)$ & $<.001$ \\
\hline $\begin{array}{l}\text { Cognitive Performance } \\
\text { Scale score }\end{array}$ & $0.94(0.92-0.97)$ & $<.001$ \\
\hline Baseline ADL score $\leq 4$ & $-9.92(-10.1-9.76)$ & $<.001$ \\
\hline Baseline ADL score $\geq 24$ & $7.26(7.15-7.36)$ & $<.001$ \\
\hline \multicolumn{3}{|l|}{$\begin{array}{l}\text { Factors that affect } \\
\text { the rate of change } \\
\text { in } \mathrm{ADL} \text { (slope) across } \\
\text { both time periods }\end{array}$} \\
\hline Time (fractions of quarters) & $-0.41(-0.46-0.36)$ & $<.001$ \\
\hline $\begin{array}{l}\text { Cognitive Performance } \\
\text { Scale score }\end{array}$ & $0.24(0.23-0.26)$ & $<.001$ \\
\hline Baseline ADL score $\leq 4$ & $1.39(1.31-1.47)$ & $<.001$ \\
\hline Baseline ADL score $\geq 24$ & $-1.09(-1.14-1.04)$ & $<.001$ \\
\hline \multicolumn{3}{|l|}{$\begin{array}{l}\text { Factors that affect ADL } \\
\text { level after hospital } \\
\text { discharge (intercept) }\end{array}$} \\
\hline Hip fracture & $7.65(7.50-7.79)$ & $<.001$ \\
\hline Stroke & $6.53(6.33-6.73)$ & $<.001$ \\
\hline Renal failure & $3.41(3.23-3.59)$ & $<.001$ \\
\hline Septicemia & $2.97(2.85-3.10)$ & $<.001$ \\
\hline Urinary tract infection & $2.75(2.62-2.87)$ & $<.001$ \\
\hline Pneumonia & $2.74(2.65-2.83)$ & $<.001$ \\
\hline Congestive heart failure & $2.64(2.49-2.79)$ & $<.001$ \\
\hline Hospital length of stay ${ }^{b}$ & $0.17(0.16-0.18)$ & $<.001$ \\
\hline \multicolumn{3}{|l|}{$\begin{array}{l}\text { Factors that affect } \\
\text { the rate of change in } \\
\text { ADL after hospital } \\
\text { discharge (slope) }\end{array}$} \\
\hline Hip fracture & $-1.39(-1.52-1.27)$ & $<.001$ \\
\hline Stroke & $0.89(0.72-1.05)$ & $<.001$ \\
\hline Renal failure & $0.58(0.42-0.74)$ & $<.001$ \\
\hline Septicemia & $0.78(0.68-0.89)$ & $<.001$ \\
\hline Urinary tract infection & $0.35(0.24-0.46)$ & $<.001$ \\
\hline Pneumonia & $0.45(0.37-0.53)$ & $<.001$ \\
\hline Congestive heart failure & $0.33(0.20-0.47)$ & $<.001$ \\
\hline Hospital length of stay & $-0.01(-0.02-0.01)$ & .33 \\
\hline
\end{tabular}

Main effect estimates are factors that affect ADL level, and time interactions are factors that affect the rate of change. For example, each point increase in Cognitive Performance Scale score was associated with an ADL score that was 0.94 points worse (higher) and also an ADL score that worsened 0.24 points per quarter, or 0.96 points per year.

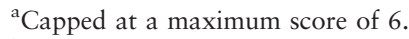

${ }^{\text {b}}$ Per day, centered on median of 5 days.

found a mean prehospital slope of -0.41 per quarter $(-0.14$ per month), indicating slow improvement. This probably represents a cohort difference. Residents with severe cognitive impairment or complete eating dependency were not excluded; if they survived, these residents 
Table 4. Parameter Estimates for the Log-Normal Regressions for Event Times Portion of the Joint Model

Effect

Multiplicative Effects (95\% Confidence Interval) $P$-Value

\begin{tabular}{lcr}
\hline Intercept & $14.0(12.0-16.2)<.001$ & $13.7(11.8-15.9)<.001$ \\
Female & $1.15(1.08-1.21)<.001$ & $1.24(1.15-1.34)<.001$ \\
Age $\geq 85$ & $0.74(0.70-0.77)<.001$ & $1.35(1.26-1.45)<.001$ \\
Cognitive Performance & $0.76(0.75-0.77)<.001$ & $1.03(1.00-1.05) .02$ \\
Scale score & & \\
Pneumonia & $1.04(0.92-1.17) .54$ & $0.50(0.44-0.57)<.001$ \\
Septicemia & $0.75(0.66-0.86)<.001$ & $0.49(0.43-0.56)<.001$ \\
Congestive heart failure & Reference & $0.28(0.24-0.32)<.001$ \\
Hip fracture & $1.98(1.68-2.33)<.001$ & Reference \\
Urinary tract infection & $1.85(1.62-2.13)<.001$ & $0.40(0.35-0.46)<.001$ \\
Renal failure & $0.90(0.76-1.06) .20$ & $0.54(0.45-0.64)<.001$ \\
Stroke & $0.33(0.28-0.40)<.001$ & $0.80(0.67-0.97) .02$ \\
Charlson Comorbidity & $0.97(0.96-0.99)<.001$ & $0.82(0.80-0.84)<.001$ \\
Index & & \\
Hospital length of stay & $0.98(0.97-0.98)<.001$ & $0.95(0.94-0.96)<.001$ \\
\hline
\end{tabular}

Because time was log-transformed, the parameter estimates were exponentiated and are interpreted as multiplicative factors on the time to event. For example, holding the other variables constant, time to readmission in quarters would be 1.24 times as long for a female resident as for a male resident (readmission would be delayed).

could only remain stable or improve. In addition, prehospital trajectories in the data were free of intervening hospital stays that would also tend to be associated with worsening ADL function.

Consistent with the results, for older adults admitted to a $\mathrm{NH}$ with disability after an acute hospitalization, another study ${ }^{44}$ found that recovering prehospital function was unlikely. In addition to better gross motor coordination and manual dexterity, that study found that intact cognition and stable weight were associated with recovery. Acute events and hospitalization in older adults appear to be periods of substantial change. Recognizing these changes should prompt practitioners to consider potential changes in care priorities after acute hospital stays.

This study has several strengths but also some limitations. A large, national cohort of long-stay NH residents was analyzed. $\mathrm{NH}$ assessments were linked to Medicare data, which provided more information on diagnoses than is available in the MDS. The timing of MDS assessments and hospital stays required many potential participants to be excluded to allow for sufficient data for meaningful analysis. Because future acute hospitalizations were unknown at the time of assessment, it is not likely that limiting the sample to residents with an assessment in the 30 days before hospital admission biased the sample. Moreover, it is likely that excluding residents who were hospitalized for fewer than 90 days before the study admission biased the results toward the positive side, if anything. Therefore, from the perspective of care planning after an acute hospitalization, these findings may provide guidance regarding consideration of more of a palliative approach for residents with an extremely poor prognosis for meaningful recovery. Although the cohort was restricted to residents hospitalized for the seven most common hospital diagnoses, these represented more than $50 \%$ of hospitalized residents and included chronic and acute conditions.

\section{CONCLUSION}

Baseline ADL status, impaired cognition, female sex, comorbid burden, hospital length of stay, and hospital diagnosis were associated with ADL decline in $\mathrm{NH}$ residents. In addition, each resident's prehospital ADL trajectory was associated with mortality and readmission, indicating that ADL trajectory is associated with other important outcomes. Finally, consistent with work in other settings, significant cognitive impairment bodes poorly for recovery from acute events. Cognitive status and ADL trajectory before an acute illness are important considerations for prioritizing care goals for residents admitted to the hospital and on their return to the NH if they survive.

This first step in modeling suggests several areas for future study. In particular, investigating the effects of facility organizational structure and care delivery on resident ADL trajectories is a logical next step. For example, do residents with hip fracture in facilities with on-site physical therapy do better than residents of facilities without these services? In addition, specifically examining the effects of multiple hospitalizations has important implications for care priorities. Further work should examine the effects of prospective efforts to reduce hospitalizations (e.g., Interventions to Reduce Acute Care Transfers $\mathrm{II}^{45}$ ) in terms of the functional status of residents. Finally, future work should prospectively evaluate whether providing information on likely functional outcomes leads to better or even different care decisions. Knowing potential outcomes could help individuals and families make clear decisions about shifting care goals. This study lays the foundation for a line of research using expected functional outcomes to inform care decisions.

\section{ACKNOWLEDGMENTS}

Preliminary results were presented at the Ninth International Conference on Health Policy Statistics, Cleveland, 
Ohio, October 2011, and the 39th North American Primary Care Research Group Annual Meeting, Banff, Alberta, Canada, November 2011.

Conflict of Interest: The editor in chief has reviewed the conflict of interest checklist provided by the authors and has determined that the authors have no financial or any other kind of personal conflicts with this paper.

Research reported in this publication was supported by the National Institute on Aging of the National Institutes of Health under Award R01AG028476. The content is solely the responsibility of the authors and does not necessarily represent the official views of the National Institutes of Health.

Author Contributions: Study concept and design: Kruse, Petroski, Mehr, Banaszak-Holl, Intrator. Acquisition of data: Kruse. Analysis and interpretation of data: Kruse, Petroski, Mehr, Banaszak-Holl, Intrator. Preparation of manuscript: Kruse, Petroski, Mehr, Banaszak-Holl, Intrator.

Sponsor's Role: None.

\section{REFERENCES}

1. Tseng SZ, Wang RH. Quality of life and related factors among elderly nursing home residents in southern Taiwan. Public Health Nurs 2001;18:304-311.

2. Covinsky KE, Wu AW, Landefeld CS et al. Health status versus quality of life in older patients: Does the distinction matter? Am J Med 1999;106:435-440.

3. Manton KG. A longitudinal study of functional change and mortality in the United States. J Gerontol 1988;43:S153-S161.

4. Gillen P, Spore D, Mor V et al. Functional and residential status transitions among nursing home residents. J Gerontol A Biol Sci Med Sci 1996;51A: M29-M36.

5. Mor V, Wilcox V, Rakowski W et al. Functional transitions among the elderly: Patterns, predictors, and related hospital use. Am J Public Health 1994;84:1274-1280.

6. Chuang KH, Covinsky KE, Sands LP et al. Diagnosis-related groupadjusted hospital costs are higher in older medical patients with lower functional status. J Am Geriatr Soc 2003;51:1729-1734.

7. Finch M, Kane RL, Philp I. Developing a new metric for ADLs. J Am Geriatr Soc 1995;43:877-884.

8. Mehr DR, Zweig SC, Kruse RL et al. Mortality from lower respiratory infection in nursing home residents: A pilot prospective community-based study. J Fam Pract 1998;47:298-304.

9. Mehr DR, Binder EF, Kruse RL et al. Predicting mortality from lower respiratory infection in nursing home residents: The Missouri LRI Study. JAMA 2001;286:2427-2436.

10. Wolinsky FD, Armbrecht ES, Wyrwich KW. Rethinking functional limitation pathways. Gerontologist 2000;40:137-146.

11. Boaz RF. Improved versus deteriorated physical functioning among longterm disabled elderly. Med Care 1994;32:588-602.

12. Creditor MC. Hazards of hospitalization of the elderly. Ann Intern Med 1993;118:219-223.

13. Boyd CM, Xue QL, Guralnik JM et al. Hospitalization and development of dependence in activities of daily living in a cohort of disabled older women: The Women's Health and Aging Study I. J Gerontol A Biol Sci Med Sci 2005;60A:888-893.

14. Sands LP, Yaffe K, Lui LY et al. The effects of acute illness on ADL decline over 1 year in frail older adults with and without cognitive impairment. J Gerontol A Biol Sci Med Sci 2002;57A:M449-M454.

15. Fried TR, Gillick MR, Lipsitz LA. Short-term functional outcomes of longterm care residents with pneumonia treated with and without hospital transfer. J Am Geriatr Soc 1997;45:302-306.

16. Binder EF, Kruse RL, Sherman AK et al. Predictors of short-term functional decline in survivors of nursing home-acquired lower respiratory tract infection. J Gerontol A Biol Sci Med Sci 2003;58A:60-67.

17. Inouye SK, Wagner DR, Acampora D et al. A predictive index for functional decline in hospitalized elderly medical patients. J Gen Intern Med 1993;8:645-652.
18. Gill TM, Allore HG, Holford TR et al. Hospitalization, restricted activity, and the development of disability among older persons. JAMA 2004;292:2115-2124.

19. Covinsky KE, Palmer RM, Fortinsky RH et al. Loss of independence in activities of daily living in older adults hospitalized with medical illnesses: Increased vulnerability with age. J Am Geriatr Soc 2003;51:451-458.

20. Jones A. The National Nursing Home Survey: 1999 summary. Vital Health Stat 13 2002;20:1-116.

21. Keeler EB, Kane RL, Solomon DH. Short- and long-term residents of nursing homes. Med Care 1981;19:363-370.

22. Intrator O, Zinn J, Mor V. Nursing home characteristics and potentially preventable hospitalizations of long-stay residents. J Am Geriatr Soc 2004;52:1730-1736.

23. Intrator O, Grabowski DC, Zinn J et al. Hospitalization of nursing home residents: The effects of states' Medicaid payment and bed-hold policies. Health Serv Res 2007;42:1651-1671.

24. Fried TR, Mor V. Frailty and hospitalization of long-term stay nursing home residents. J Am Geriatr Soc 1997;45:265-269.

25. Stuck AE, Walthert JM, Nikolaus T et al. Risk factors for functional status decline in community-living elderly people: A systematic literature review. Soc Sci Med 1999;48:445-469.

26. Brooks S, Warshaw G, Hasse L et al. The physician decision-making process in transferring nursing home patients to the hospital. Arch Intern Med 1994;154:902-908.

27. Scott HD, Logan M, Waters WJ Jr et al. Medical practice variation in the management of acute medical events in nursing homes: A pilot study. R I Med J 1988;71:69-74.

28. Morrison RS, Siu AL. Survival in end-stage dementia following acute illness. JAMA 2000;284:47-52.

29. Anderson RT, James MK, Miller ME et al. The timing of change: Patterns in transitions in functional status among elderly persons. J Gerontol B Psychol Sci Soc Sci 1998;53B:S17-S27.

30. Fortinsky RH, Covinsky KE, Palmer RM et al. Effects of functional status changes before and during hospitalization on nursing home admission of older adults. J Gerontol A Biol Sci Med Sci 1999;54A:M521-M526.

31. Phillips CD, Morris JN, Hawes C et al. Association of the Resident Assessment Instrument (RAI) with changes in function, cognition, and psychosocial status. J Am Geriatr Soc 1997;45:986-993.

32. Walk D, Fleishman R, Mandelson J. Functional improvement of elderly residents of institutions. Gerontologist 1999;39:720-728.

33. Zuliani G, Romagnoni F, Volpato $S$ et al. Nutritional parameters, body composition, and progression of disability in older disabled residents living in nursing homes. J Gerontol A Biol Sci Med Sci 2001;56A:M212-M216.

34. Kurella Tamura M, Covinsky KE, Chertow GM et al. Functional status of elderly adults before and after initiation of dialysis. $\mathrm{N}$ Engl J Med 2009;361:1539-1547.

35. Morris JN, Fries BE, Morris SA. Scaling ADL's within the MDS. J Gerontol A Biol Sci Med Sci 1999;54A:M546-M553.

36. Morris JN, Fries BE, Mehr DR et al. MDS cognitive performance scale. J Gerontol 1994;49:M174-M182.

37. Murphy TE, Han L, Allore HG et al. Treatment of death in the analysis of longitudinal studies of gerontological outcomes. J Gerontol A Biol Sci Med Sci 2011;66A:109-114.

38. Henderson R, Diggle P, Dobson A. Joint modelling of longitudinal measurements and event time data. Biostatistics 2000;1:465-480.

39. Guo X, Carlin BP. Separate and joint modeling of longitudinal and event time data using standard computer packages. Am Stat 2004;58:16-24.

40. Lancaster T, Intrator O. Panel data with survival: Hospitalization of HIVpositive patients. J Am Stat Assoc 1998;93:46-53.

41. Sager MA, Rudberg MA, Jalaluddin M et al. Hospital Admission Risk Profile (HARP): Identifying older patients at risk for functional decline following acute medical illness and hospitalization. J Am Geriatr Soc 1996;44:251-257.

42. McConnell ES, Pieper CF, Sloane RJ et al. Effects of cognitive performance on change in physical function in long-stay nursing home residents. J Gerontol A Biol Sci Med Sci 2002;57A:M778-M784.

43. Charlson ME, Pompei P, Ales KL et al. A new method of classifying prognostic comorbidity in longitudinal studies: Development and validation. J Chronic Dis 1987;40:373-383.

44. Gill TM, Gahbauer EA, Han L et al. Factors associated with recovery of prehospital function among older persons admitted to a nursing home with disability after an acute hospitalization. J Gerontol A Biol Sci Med Sci 2009;64A:1296-1303.

45. Ouslander JG, Lamb G, Tappen $R$ et al. Interventions to reduce hospitalizations from nursing homes: Evaluation of the INTERACT II collaborative quality improvement project. J Am Geriatr Soc 2011;59:745-753. 


\section{SUPPORTING INFORMATION}

Additional Supporting Information may be found in the online version of this article:

Appendix S1. Statistical appendix for ADL trajectories surrounding acute hospitalization of long-stay nursing home residents.
Please note: Wiley-Blackwell is not responsible for the content, accuracy, errors, or functionality of any supporting materials supplied by the authors. Any queries (other than missing material) should be directed to the corresponding author for the article. 